\title{
BRAIDED THREADS WITH AC ELECTROSPUN NANOFIBERS FOR HYGIENIC AND MEDICAL APPLICATIONS - PRODUCTION AND PROPERTIES
}

\author{
Divyabharathi MADHESWARAN ${ }^{1}$, Šárka HAUZEROVÁ ${ }^{1}$, Jan VALTERA ${ }^{1}$, Maxim LISNENKO ${ }^{1}$, \\ Manikandan SIVAN ${ }^{1}$, Ondřej BAŤKA ${ }^{1}$, Věra JENČOVÁ ${ }^{1}$, David LUKÁŠ ${ }^{1}$, František JANDA ${ }^{2}$, \\ Eva KUŽELOVÁ KOŠŤÁKOVÁ ${ }^{1}$ \\ ${ }^{1}$ Technical University of Liberec, Liberec, Czech Republic, EU, divyabharathi.madheswaran@tul.cz \\ ${ }^{2}$ Wikinomist, s.r.o.
}

https://doi.org/10.37904/nanocon.2021.4355

\begin{abstract}
Threads are the basic textile linear structure used in many areas. The variability of the thread structure made possible by special constructions brings great possibilities of variability. It is also possible to introduce nanofiber material into the yarn construction as a cover for central microfiber yarns, and these can be further intertwined into more complex yarn constructions. Continuous production of so-called composite yarns containing central microfiber yarns and nanofiber wrappers is made possible by alternating current electrospinning. Subsequent braiding of these composite yarns allows the protection of nanofiber packages and the variability of the overall construction of the resulting yarns. This paper introduces the creation of yarns and various types of basic as well as braided yarns with nanofibers. Selected sutures were further subjected to in-vitro testing to verify cytocompatibility for application in hygiene such as dental sutures or medicine as surgical sutures.
\end{abstract}

Keywords: AC electrospinning, composite nanofibrous yarns, braiding, cytocompatibility, dental floss

\section{INTRODUCTION}

Electrospinning is one of the widely used techniques for the fabrication of polymer-based nanofibers $[1,2]$. Usually, this technology uses a DC high voltage source to create nanofibrous planner materials [3]. However, for the formation of linear structures, i.e., nanofibrous yarns or composite yarns with a nanofibrous sheath wounded around the classic yarn, it is facile to use an alternating current voltage (AC) source over DC source. During the AC electrospinning process [4], the emerging fibrous plume from the AC electrode can be deposited on an electrically inactive substrate due to the $A C$ nature of the power source. Hence it is much easier to collect the nanofibers on the classic yarns to construct the core-nanofibrous sheath structure using $A C$ electrospinning. In this work, polycaprolactone (PCL) and polyvinyl alcohol (PVA) have been selected due to their excellent biocompatibility and electrospinnability. In addition, low toxic solvents were used to prepare the ideal spinning solutions [5] of PCL or PVA. Composite yarns consisting of a micro-fibrous core and nanofibrous shell (PCL or PVA) can be used in medical or hygiene applications. Despite the inherent biocompatibility of the polymer, the cytotoxicity of the end product needs to be analysed. Hence, the objective of the following study is to prepare PCL or PVA nanofibrous sheath-core (PA6) composite yarn using the combinations of AC electrospinning and braiding technology. Subsequently, we examined the cytotoxicity of the composite yarns and braided composite yarns.

\section{MATERIALS AND METHODS}

For the composite yarn production, core yarn in multifilament form from polyamide 6 (PA6) twelve trilobal fibers with linear density 44 dtex (Odetka a.s. CZ) was used. The nanofibrous shell was produced from polycaprolactone (PCL; $M_{n} 80.000$ ) or polyvinyl alcohol (PVA; $\left.M_{n} 125.000\right)$, both purchased from Merck $C Z$. 
Acetic acid, formic acid, acetone, and HPLC grade water were obtained from Penta CZ. Polymeric solutions were prepared by dissolving the PCL pellets in acetic acid/formic acid/acetone (1/1/1 v/v/v) and PVA in HPLC grade water to prepare $10 \mathrm{wt} \%$ solutions of PCL and PVA, respectively.

The fabrication of composite nanofibrous yarn using industrial-scale AC electrospinning is shown in Figure1. This device was designed and constructed at the Technical University of Liberec using the following components: 1) high voltage AC power source, 2) solution reservoir which was embedded with screw pump and electrode, and 3 ) continuously running core yarn (as a collector), 4) twirling devices and 5) heating zones (for removing the residual solvents) [6,7]. The distance between the spinning electrode and the core yarn was set to $250 \mathrm{~mm}$. As mentioned earlier, the moving core yarn was used as an electrically inactive collector for capturing the emerging nanofibrous plume from the electrode. The feeding rate of the core yarn or production rate of the composite yarn was maintained at $10 \mathrm{~m} / \mathrm{min}$. From each material, $200 \mathrm{~m}$ of composite nanofibrous yarn was produced. All the resultant composite yarns were fabricated using the applied effective AC high voltage of $34.5 \mathrm{kV}$. Furthermore, these composite yarns were subsequently braided using 8 or 9 individual composite yarns from each material. It is worth mentioning that the braiding machine was slightly modified in order to render the damage to the nanofibrous envelope.

The Linear density of the materials (pristine and braided) was calculated in Decitex using the following formula (mass of the yarn/length of the yarn) $\times 10000$ and corresponding results have been listed in Table 1 .

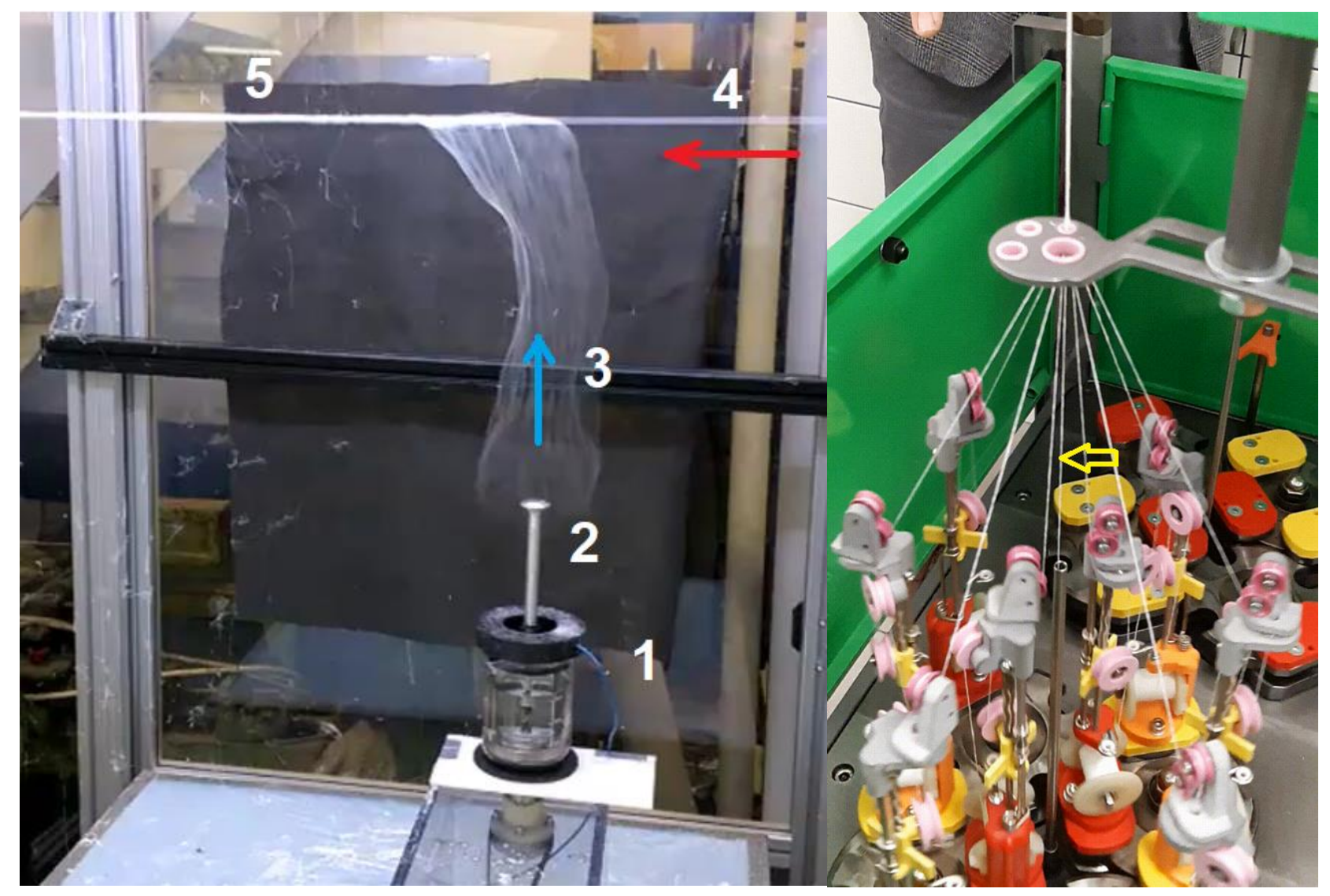

Figure 1 Photo of composite yarn production by AC electrospinning (left): 1 - connection to an AC high voltage source; 2 - spinning electrode with a polymer solution and feeding system; 3 - emerging nanofibers; 4 - feeding the core yarn; 5 - withdrawing the resulting composite yarn. Photo of detail to braiding zone, in braiding machine during braiding of 9 yarns (the middle yarn indicated by the arrow). 
Surface morphology of the composite (Figure 2) and braided yarn (Figure 3) were evaluated using TESCAN Vega 3 (Czech Republic) scanning electron microscopy (SEM) and UHR-FE-SEM Zeiss Ultra Plus Microscope (Germany). Prior to SEM analysis, all the samples were gold coated.

Evaluation of the cytotoxicity of the materials was tested (in-vitro) with the cell line 3T3-A31 mouse fibroblasts (ATCC). The standard determines the material as cytotoxic if the viability of cells incubated with the extracts of the monitored material in the culture medium reaches values lower than $70 \%$ viability of negative control cells (cells incubated in the complete medium=DMEM) [8].

The cells were incubated in complete culturing medium DMEM High Glucose (Dulbecco's Modified Engles Medium, Merck) with 10\% Fetal bovine serum (Biosera) and 1\% antibiotic - Pen / Strep Amphotericin B (Lonza). For testing, the sterilized samples (using ethylene oxide) with three different lengths were prepared from each material which were 2, 4, and $6 \mathrm{~cm}$. The samples were placed in the 96 well plates, and subsequently, a glass ring was placed on the sample to fix at the bottom of the well.

On the first day, cells were seeded in 96 wells plates (passage 19). The concentration of cells suspension was 10000 cells per $100 \mu \mathrm{l}$ (per one well). For preparing the extracts of the sample, $1 \mathrm{ml}$ of complete culture medium was added to the sample-loaded well plates (10 wells/sample). The well plates with cells and samples were incubated at $37{ }^{\circ} \mathrm{C}$ in a $\mathrm{CO}_{2}$ incubator for 24 hours. On the second day, the culture medium from the cellseeded well plate was removed, and subsequently, $100 \mu \mathrm{l}$ of an extract from the samples was added and then incubated for $24 \mathrm{hrs}$ in a $\mathrm{CO}_{2}$ incubator. On the third day, the extract from the wells with cells was removed, and subsequently, $100 \mu \mathrm{l}$ of MTT solution was added and incubated at $37^{\circ} \mathrm{C}$ in the $\mathrm{CO}_{2}$ incubator for 2 hours. After incubation, the MTT solution was removed, and add $100 \mu \mathrm{l}$ of isopropyl alcohol and mixed by pipette. The UV absorbance was measured at the wavelength of $470 \mathrm{~nm}$ (reference was $650 \mathrm{~nm}$ ) by spectrophotometer (Tescan).

\section{RESULTS AND DISCUSSION}

The polymeric solutions of PCL and PVA were spinnable using the AC electrospinning. In addition, linear density analysis of the samples reveals that PCL-based composite and braided yarns result in better spinnability than PVA (Table 1). From the SEM images (Figure 2), it is thus clear that PCL and PVA-based electrospun nanofiber were completely wounded around the PA6 core yarn. Moreover, the utilized braiding process as well did not damage the nanofibrous envelope of the braided composite yarns, which was also seen from the SEM images (Figure 3). Hence, the SEM and linear density analysis of the composite and braided composite yarn shows that the prime objective of the following study was successfully achieved in terms of fabricating the composite and braided composite yarn without damaging the nanofibrous envelope.

Table 1 Overview of the tested yarns with their final linear densities

\begin{tabular}{|l|c|}
\hline \multicolumn{1}{|c|}{ Sample } & $\begin{array}{c}\text { Linear density } \\
\text { [dtex] }\end{array}$ \\
\hline PA6 core yarn & $46 \pm 0.7$ \\
\hline PA6 8 braided yarns & $362 \pm 0.7$ \\
\hline PA6/PCL composite yarn & $112.6 \pm 0.5$ \\
\hline PA6/PCL 8 braided composite yarns & $1040 \pm 1.1$ \\
\hline PA6/PCL 9 braided composite yarns & $1121.8 \pm 1.6$ \\
\hline PA6/PVA composite yarn & $70.6 \pm 1.5$ \\
\hline PA6/PVA 8 braided composite yarns & $563 \pm 2$ \\
\hline
\end{tabular}




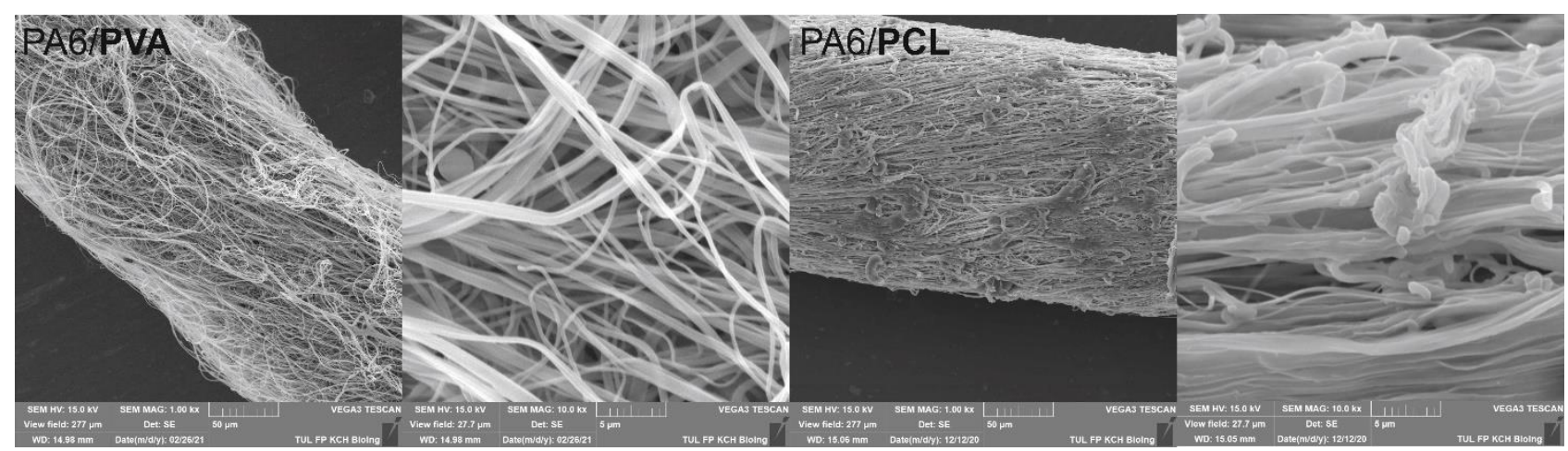

Figure 2 SEM images of the surface of composite yarns with AC electrospun nanofibrous sheath from PVA (left) and PCL (right)
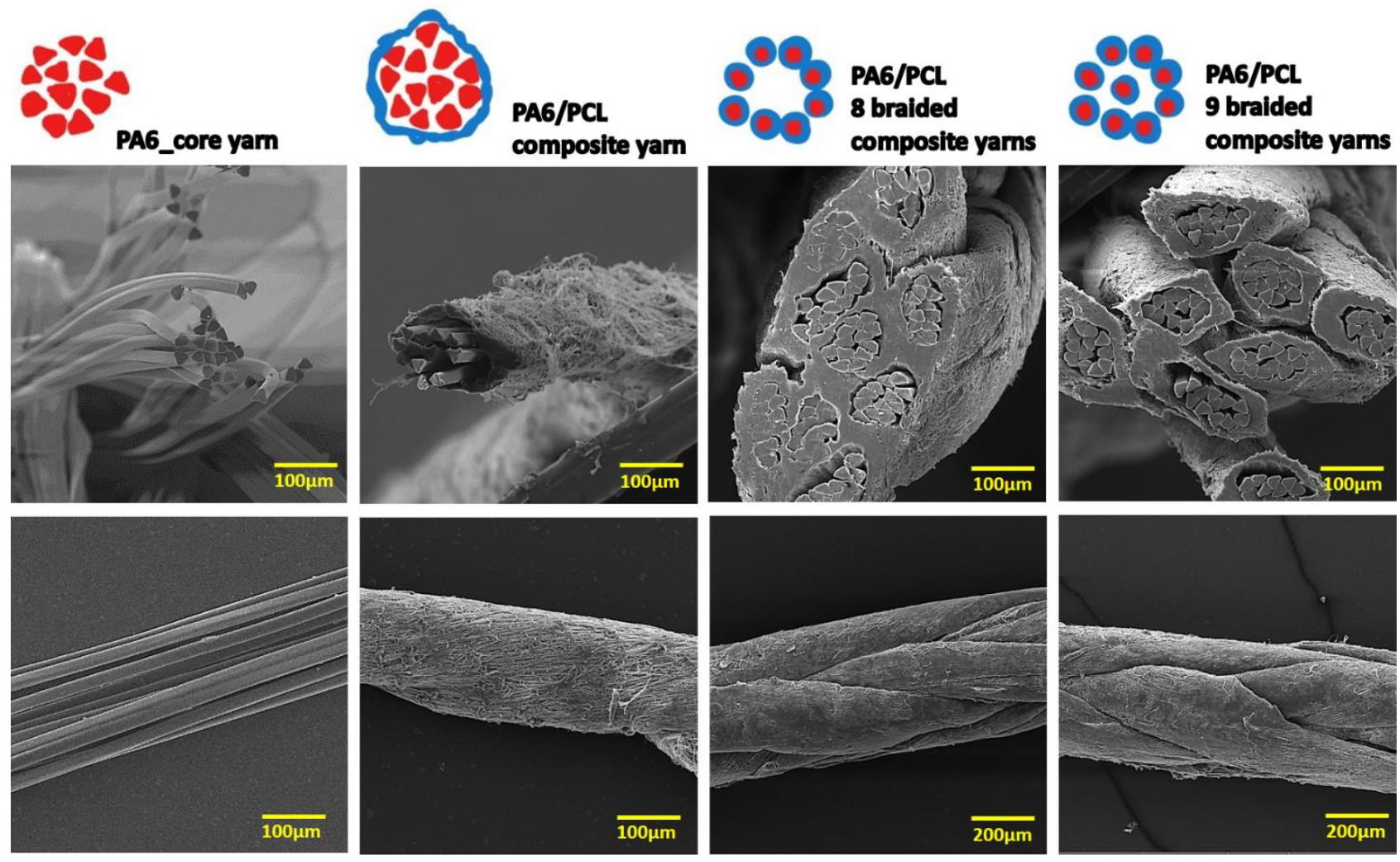

Figure 3 Scheme (above) and SEM images (cross-section view in middle and longitudinal view bellow) of microfibrous multifilament core yarn (PA6) and composite yarn (PA6/PCL) and braided composite yarns from eight and nine composite yarns

It is well known that the PCL and PVA were biocompatible. However, the MTT metabolic assay was performed whether the used polymer processing technologies (solution, AC electrospinning, and braiding) is induced any cytotoxicity on the fabricated materials. The outcomes of the MTT assay were evaluated according to the standard ČSN EN ISO 10993-5 (85 5220), and the results are shown in Figure 4. All the fabricated samples were not shown cytotoxic effects on the 3T3-SA mouse fibroblasts cell with more than $80 \%$ cell viability. In that, $2 \mathrm{~cm}$ length of all the samples were shown higher cell viability than that of 4 and $6 \mathrm{~cm}$. It could be attributed to the leaching of the small amount of residual solvent from the length of the $2 \mathrm{~cm}$ sample. In contrast, a higher sample length may leach out a probably higher amount of residual solvents, which may slightly reduce the viability of the cells. Thus, the MTT assay shows that all the fabricated and braided composite yarn was 
biocompatible with the 3T3-SA mouse fibroblasts cell. Adhesion and proliferation of the cells on these materials for a more extended time will be evaluated in the future. Due to its biocompatibility, these samples could be used as dental threads or surgical sutures in medical fields.

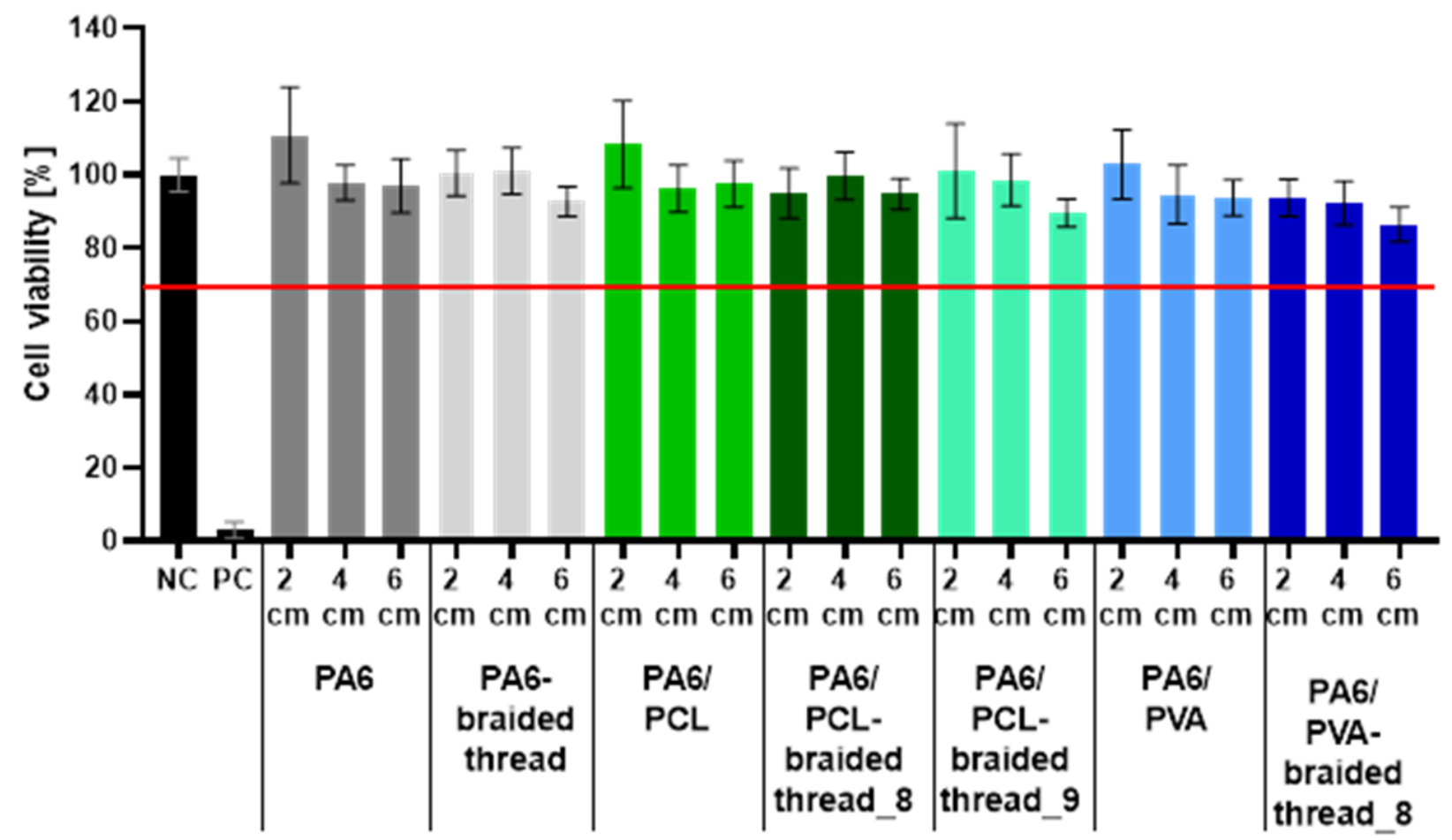

\section{Materials}

Figure 4 Bar graph of cell viability measured by metabolic assay (MTT) after culturing 3T3-SA mouse fibroblasts with the extract of pristine core yarn, composite nanofibrous yarns, and braided composite yarns. From each material were tested three different lengths $(2 \mathrm{~cm}, 4 \mathrm{~cm}$, and $6 \mathrm{~cm}) . \mathrm{NC}$ is negative control and PC positive control.

\section{CONCLUSION}

The article presented the manufacturing and testing of composite yarns and braided composite yarns formed by a microfiber core and a nanofiber sheath in several material combinations. The resultant yarns were subsequently tested for possible cytotoxicity or cytocompatibility through cell viability testing using metabolic MTT assay. The results of the tests significantly show that the technological steps of the production of composite yarns and the subsequent braiding did not bring any cytotoxic effect to the resulting yarns; hence all the materials were biocompatible. Therefore, the fabricated materials can be considered as suitable carriers for specific active substances according to specific applications, such as surgical sutures or dental flosses in the medical field.

\section{ACKNOWLEDGEMENTS}

This work was supported by the project Technology Agency of the Czech Republic TACR Trend No. FW02020042. 


\section{REFERENCES}

[1] BARHOUM, A., PAL, K., RAHIER, H., ULUDAG, H., KIM, I. S., BECHELANY, A M. Nanofibers as new-generation materials: From spinning and nano-spinning fabrication techniques to emerging applications. Applied Materials Today. 2019, vol. 17, pp. 1-35. Available from: https://doi.org/10.1016/j.apmt.2019.06.015.

[2] KENRY, LIM, C. T. Nanofiber technology: current status and emerging developments. Progress in Polymer Science. 2017, vol. 70, pp. 1-17. Available from: https://doi.org/10.1016/j.progpolymsci.2017.03.002.

[3] LIN, T., WANG, A X. Needleless electrospinning of nanofibers: technology and applications. Singapore: Pan Stanford Publications, 2014.

[4] POKORNY, P. et al. Effective AC needleless and collectorless electrospinning for yarn production. Phys. Chem. Chem. Phys. 2014, vol. 16, no. 48, pp. 26816-26822. Available from: https://doi.org/10.1039/C4CP04346D.

[5] SIVAN, M. et al. Plasma treatment effects on bulk properties of polycaprolactone nanofibrous mats fabricated by uncommon AC electrospinning: A comparative study. Surface and Coatings Technology. 2020, vol. 399, p. 126203. Available from: https://doi.org/10.1016/j.surfcoat.2020.126203.

[6] VALTERA, J. et al. Fabrication of dual-functional composite yarns with a nanofibrous envelope using high throughput AC needleless and collectorless electrospinning. Sci Rep.2019, vol. 9, no. 1, p. 1801. Available from: https://doi.org/10.1038/s41598-019-38557-z.

[7] AMLER, E. et al. Method for Production of Polymeric Nanofibers by Spinning of Solution or Melt of Polymer in Electric Field, and a Linear Formation from Polymeric Nanofibers Prepared by This Method. 2014. WO2014094694A1.

[8] HRAZDIL, J., RYCHNOVSKY, A L. Biological evaluation of medical devices - Part 1: Evaluation and testing; ČSN EN ISO 10993-1. Český normalizační institut. [Online]. 2004. [Accessed 13 July 2021]. Available from: https://doi.org/http://csnonlinefirmy.agentura-cas.cz/html nahledy/85/70508/70508 nahled.htm 